\title{
Insulin-like growth factor I acts as an angiogenic agent in rabbit cornea and retina: comparative studies with basic fibroblast growth factor
}

\author{
M.B.Grant ${ }^{1,2}$, R.N.Mames ${ }^{2}$, C. Fitzgerald ${ }^{2}$, E. A.Ellis ${ }^{2}$, M. Aboufriekha ${ }^{1}$, J. Guy ${ }^{2}$ \\ ${ }^{1}$ Department of Medicine, University of Florida, Gainesville, Florida, USA \\ ${ }^{2}$ Department of Ophthalmology, University of Florida, Gainesville, Florida, USA
}

\begin{abstract}
Summary. The release of growth factors from ischaemic retina has been hypothesized as the central stimulus for retinal neovascularization in proliferative diabetic retinopathy. Two of the growth factors implicated are insulin-like growth factor-I and basic fibroblast growth factor. We examined the effect of insulin-like growth factor-I on in vivo neovascularization using the established angiogenic model of the rabbit cornea $(n=30)$, and also compared the effects of insulin-like growth factor-I and basic fibroblast growth factor using two new in vivo systems. Either supraphysiologic concentrations of each growth factor $(600 \mu \mathrm{g})$ were injected intravitreally into pigmented rabbits $(n=21)$ or porous polyfluorotetraethylene chambers filled with an emulsion containing collagen and growth factor $(500 \mathrm{ng})$ were placed on the retina surface $(n=8)$. Our results demonstrate that when insulinlike growth factor-I was implanted together with a slow release carrier into the pocket of the normally avascular cornea, insulin-like growth factor-I $(10 \mu \mathrm{g} /$ pellet $)$ induced angiogenesis in all rabbits. This degree of angiogenesis was comparable to that previously shown for basic fibroblast
\end{abstract}

growth factor. For the intravitreal studies, the fibrotic component was greater in the basic fibroblast growth factor injected eyes, whereas the vascular component was accentuated in the eyes injected with insulin-like growth factor-I. Light and electron microscopy demonstrated areas of vascular proliferation in both groups. Porous polyfluorotetraethylene chamber studies with insulin-like growth factor-I and basic fibroblast growth factor demonstrated vascular proliferation in the vicinity of the chamber similar to the intravitreal injected eyes, but to a lesser degree than the injected eyes. Our experiments overall support the angiogenic potential of both insulin-like growth factor-I and basic fibroblast growth factor and support distinct but complimentary roles for each growth factor in the pathogenesis of proliferative diabetic retinopathy.

Key words: Insulin-like growth factor-I, basic fibroblast growth factor, angiogenesis, diabetic retinopathy, animal models.
Following the development of ischaemia in the diabetic eye, the retina responds by attempting to revascularize the ischaemic areas. This leads to the formation of new and aberrant vasculature on the surface of the retina. The responsible factors for the development of this retinal neovascularization remain to be established; however, two factors that have been implicated are insulin-like growth factor I (IGF-I) and basic fibroblast growth factor (bFGF).

Evidence suggesting a role for IGF-I in the pathogenesis of diabetic retinopathy stems from the long accepted involvement of growth hormone (GH) [1-3]. IGF-I mediates most of the mitogenic actions of GH. Although most diabetic patients have normal serum levels of IGF-I [4], elevated levels of IGF-I occur in subjects with rapidly accelerating proliferative retinopathy [5]. After controlling for glycosylated haemoglobin, blood pressure, protein- uria, duration of disease, and age at diagnosis, a large population-based study of 928 diabetic patients found higher levels of IGF-I associated with an increased frequency of proliferative diabetic retinopathy [6]. Hyer and co-workers [7] demonstrated that in the subgroup of patients with preproliferative retinopathy that subsequently developed retinal neovascularization, serum IGF-I levels at the time of the first appearance of new vessels were higher than those measured 3 months prior to the development of neovascularization [7]. Regional levels of IGFI may be more important than systemic levels. Intravitreal IGF-I concentrations have been found to be increased in diabetic patients undergoing therapeutic vitrectomy [8]. In vitro studies also support a role for IGF-I in the pathogenesis of retinal neovascularization by demonstrating that IGF-I acts at all the stages required for new vessel formation including endothelial cell proliferation [9], migra- 
tion [10] and production of proteases relevant to basement membrane degradation [11-13].

Basic fibroblast growth factor also appears to play a role in the pathogenesis of diabetic retinopathy [14-16]. Elevated concentrations of $b$-FGF have been found in vitrectomy samples from patients with proliferative diabetic retinopathy and vitreous haemorrhage [15]. b-FGF induces endothelial cell proliferation [17-19] and migration [20]. b-FGF induces the release of collagenase and plasminogen activators [21]. Although it is apparent that both IGF-I and b-FGF can act in vitro, on processes relevant to neovascularization, their ability to induce neovascularization in vivo remains to be further explored.

Basic fibroblast growth factor has previously been shown to induce corneal neovascularization $[22,23]$. We, therefore, asked whether IGF-I could induce corneal neovascularization. Because corneal neovascularization is more representative of the angiogenesis associated with wound healing, we were concerned that the retinal vasculature may respond to an angiogenic stimuli in a different manner than the cornea. We, therefore, developed two new models for specifically investigating retinovascular proliferation. The first model involved intravitreal injections of each growth factor, and the second model required the placement of slow release chambers containing each growth factor directly on the retinal surface.

\section{Materials and methods}

Preparation of the Elvax 40 slow release form polymer containing $I G F-I$. Elvax pellets (DuPont de Nemours and Co., Wilmington, Del., USA) were prepared by the method of Langer and Folkman [24]. Briefly, ten Elvax 40 pellets were washed extensively with distilled water (10 volume) followed by $97 \%$ ethanol ( 10 volume) and then with absolute ethanol until the optical density of the wash was below 0.01 . After washing, the pellets were dessicated to remove residual ethanol. The Elvax 40 was then dissolved in methylene chloride to a final concentration of $10 \%$ (weight/volume). The Elvax solution $(200 \mu \mathrm{l})$ was placed on a chilled glass surface and $20 \mu \mathrm{l}$ of methylene chloride containing either 10,50 , or $100 \mu \mathrm{g}$ of recombinant human (rh) IGF-I (Chiron Corporation, Emeryville, Calif., USA) was stirred rapidly into the Elvax 40 . The thin transparent film was gently rolled into a uniform log shape and cut into 10 identical pieces of $1 \mathrm{~mm}^{2}$ using a scalpel. Each implant contained either 1,5 , or $10 \mu \mathrm{g}$ of protein. Implants containing either 1,5 , or $10 \mu \mathrm{g}$ of bovine serum albumin (BSA) or $1 \mu \mathrm{g}$ prostaglandin $\mathrm{E}_{2}\left(\mathrm{PGE}_{2}\right)$ were made in an identical fashion for use as negative and positive controls, respectively. ${ }^{125} \mathrm{I}-\mathrm{IGF}-\mathrm{I}(266.7 \mu \mathrm{g} / \mu \mathrm{Ci})$ for use in Elvax pellets and porous polyfluorotetraethylene chambers (PPC) was prepared as previously described [8].

Implantation of pellet containing IGF-I. Implantation of the pellets was performed by the method of Gimbrone et al. [25]. The rabbits were anaesthetized with an $\mathrm{i}$. $\mathrm{m}$. injection of $30 \mathrm{mg}$ ketamine hydrochloride/ $\mathrm{kg}$ body weight and $5 \mathrm{mg}$ xylazine $/ \mathrm{kg}$ body weight. Using a no. 11 Beaver blade, a superficial incision ( $1.5 \mathrm{~mm}$ long) was made in the corneal dome to one side of its centre, and continued down approximately $2 \mathrm{~mm}$. Using a malleable iris spatula, an oblong pocket was fashioned within the corneal stroma, which ended 2-3 $\mathrm{mm}$ from the limbus. The implant containing either IGF-I, BSA, or PGE $E_{2}$ was deposited in the bottom of each pocket, which then sealed spontaneously. Eyes with corneal implants were examined daily with a Zeiss (Hanover, Md., USA) Slit-Lamp Stereomicroscope. New vessel growth was measured with an ocular micrometer at a magnification of $16 x$

A positive response was observed when capillaries in the shape of hairpin loops (weak response) or sprouting as a dense brush bor- der (strong response) grew from the adjacent limbal plexus toward the implant. Sustained growth of well-defined individual capillaries from the limbus toward or into the implant was considered positive for angiogenesis. Angiogenic response was scored from projected $35-\mathrm{mm}$ slides on a scale of $0-4$ by two independent observers who had no prior knowledge of the sample identity.

A negative response (scored zero) was observed when a few capillaries in the shape of hairpin loops developed during the first few days but later regressed. Eyes that demonstrated positive angiogenesis were enucleated on days 4, 7,14 and 21 , embedded in paraffin, sectioned, and stained with haematoxylin and eosin for histologic examination to exclude angiogenesis due to the influx of inflammatory cells.

Intravitreal injection study. Twenty-one pigmented rabbits weighing $1.4-2.5 \mathrm{~kg}$ were anaesthetized with an $1 . \mathrm{m}$. injection of $30 \mathrm{mg}$ ketamine hydrochloride/kg body weight and $5 \mathrm{mg}$ xylazine $/ \mathrm{kg}$ body weight. Their pupils were dilated with $1 \%$ topical tropicamide and $2.5 \%$ phenylephrine. Each animal was placed beneath an operating microscope, and a 30-gauge needle was inserted into the anterior chamber of one eye to withdraw $0.08 \mathrm{ml}$ of fluid. Then a 30 -gauge needle was inserted through the sclera and retina $4 \mathrm{~mm}$ posterior to the corneoscleral junction in the superotemporal quadrant. Using a stereomicroscope, $0.1 \mathrm{ml}$ of balanced salt solution containing $600 \mu \mathrm{g}$ of either IGF-I $(n=8)$ or b-FGF $(n=3)$ was injected with the bevel of the needle directed up directly over the optic nerve head. The injection was made close to the retinal surface to disperse the growth factor solution as close to the retinal surface as possible without causing a retinal tear. The rabbit was then placed supine for 30 60 min to facilitate settling of the growth factor solution onto the vascularized medullary ray and disc. Control animals received either $600 \mu \mathrm{g}$ of heat-inactivated IGF-I $(n=4), 600 \mu \mathrm{g}$ of BSA $(n=4)$ or balanced salt solution $(n=6)$. IGF-I was heat inactivated by boiling at $100^{\circ} \mathrm{C}$ for $30 \mathrm{~min}$.

All animals were examined pre-operatively and post-operatively using indirect ophthalmoscopy, fundus photography, and fluorescein angiography. All 21 rabbits were examined and photographed on days $2,4,6,8,10,12,14$, and 21 . Fundus photographs and fluorescein angiograms were graded by two independent readers who had no prior knowledge of whether the study material was from a treated or control eye.

Preparation of polyfluorotetraethylene chambers containing either $I G F-I$ or $b-F G F$. Each chamber (PPC) consisted of a $1 \times 6 \mathrm{~mm}$ tube of expanded polyfluorotetraethylene (W.L.Gore and Associates, Inc., Flagstaff, Az., USA) with a nominal pore size of $90 \mu \mathrm{m}$. The chambers were filled with an emulsion of $500 \mathrm{ng}$ of either IGF-I or b-FGF and collagen or collagen alone under sterile conditions, and using a stereomicroscope both ends of each chamber were tied with suture. Each chamber contained approximately $10 \mu \mathrm{l}$ of the collagen-growth factor mixture. For implantation of these chambers, the animal was anaesthetized and positioned beneath the operating microscope. Following removal of $0.08 \mathrm{ml}$ of aqueous from the anterior chamber, the PPC was advanced through the sclerotomy site and positioned overlying the medullary ray.

Animals implanted with chambers were examined pre-operatively and post-operatively using indirect ophthalmoscopy and fundus photography on the identical schedule as the rabbits receiving intravitreal injections.

Histologic studies of eyes undergoing either intravitreal injections or placement of $P P C$. Eyes were enucleated and small slits were made at the scleral-limbal junction prior to placing in Trump's fixative [26]. Collates cut along the medullary rays to include the optic disc were washed in potassium phosphate-sodium hydroxide buffer and postfixed overnight in $1 \%$ buffered osmium tetroxide. Specimens were dehydrated through an ethanol series to propylene oxide, infiltrated, and embedded in epoxy resin. Light microscopy sections $(2 \mu \mathrm{m}$ thick) were stained with $1 \%$ toluidine blue. Ultrathin sections were post-stained with $2 \%$ aqueous uranyl acetate followed by Reynolds lead citrate [27] and examined and photographed by transmission electron microscopy at an accelerating voltage of 75 kilo volts. 

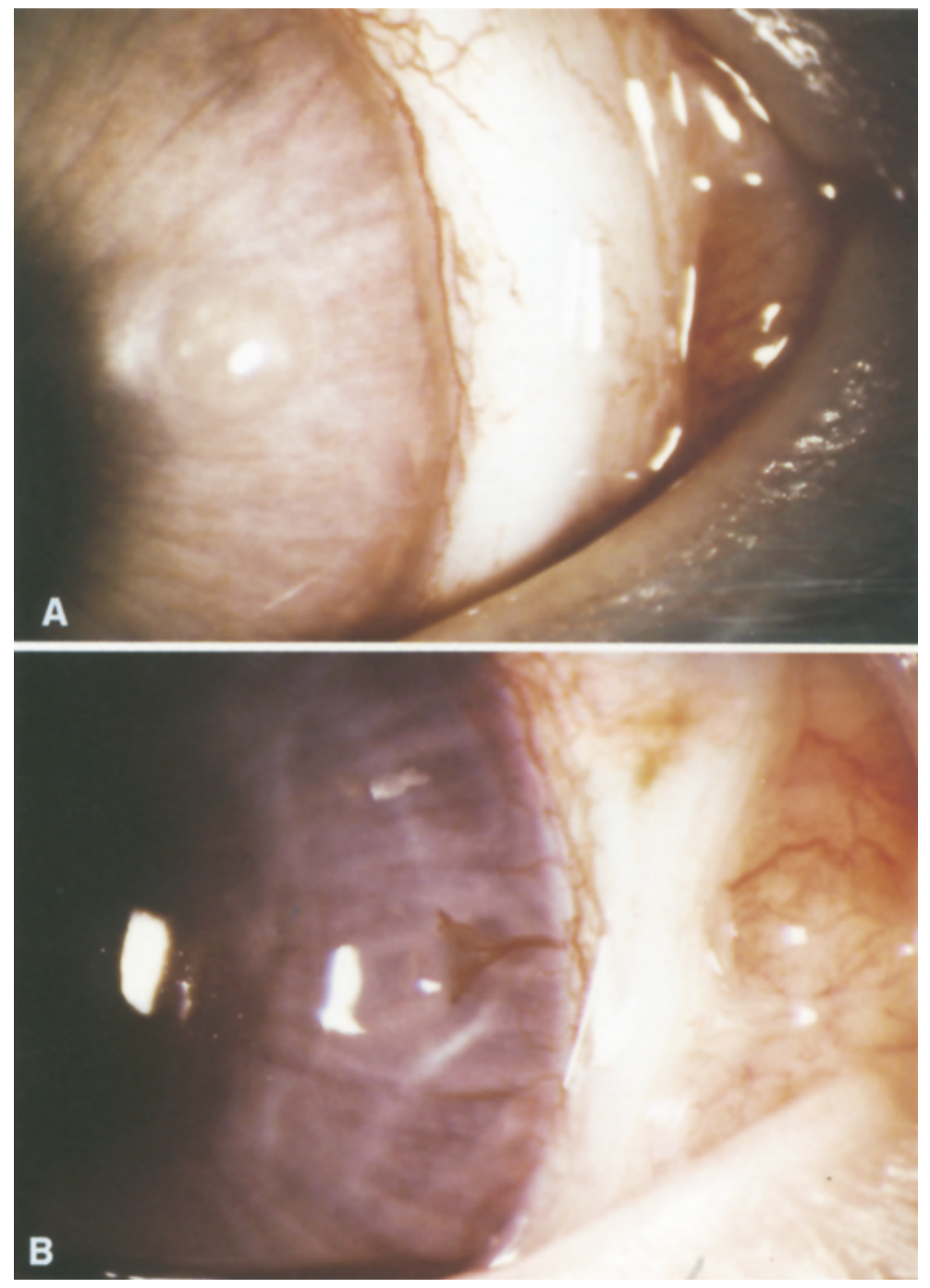

Fig. 1. A Control pellet containing albumin is shown 14 days after implantation in the rabbit cornea. There is no response by the cornea.

B Insulin-like growth factor I (IGF-I) $(10 \mu \mathrm{g})$ was placed in Elvax pellets and implanted into a rabbit corneal pouch. IGF-I demonstrates a positive response

\section{Results}

Corneal pocket studies. The corneas of 30 rabbits were implanted with control pellets containing either $1 \mu \mathrm{g}$, $5 \mu \mathrm{g}$, or $10 \mu \mathrm{g}$ of albumin. Three of 30 corneas developed a positive response; whereas, the remaining rabbits had a negative response as shown in Figure $1 \mathrm{~A}$. Five rabbits were implanted with pellets containing $1 \mu \mathrm{g}$ of IGF-I. These rabbits exhibited a negative neovascular response. Five rabbits were implanted with pellets containing $5 \mu \mathrm{g}$ of IGF-I. Only three of these rabbits exhibited a positive response. In marked contrast, all 20 rabbits implanted

Table 1. Corneal neovascularization in response to test substances in Elvax pellets implanted in rabbits

\begin{tabular}{lllll}
\hline & \multirow{2}{*}{$\begin{array}{c}\text { Number of } \\
\text { implants }\end{array}$} & \multicolumn{3}{c}{ Corneal response } \\
\cline { 3 - 5 } & & Positive & Negative & $\%$ Positive \\
\hline Control (Albumin) & 30 & 3 & 27 & 10 \\
Insulin-like growth & & & & \\
factor I & & & & \\
$1 \mu \mathrm{g}$ & 5 & 0 & 5 & 0 \\
$5 \mu \mathrm{g}$ & 5 & 3 & 2 & 60 \\
$10 \mu \mathrm{g}$ & 20 & 20 & 0 & 100 \\
\hline
\end{tabular}

with pellets containing $10 \mu \mathrm{g}$ of IGF-I developed a positive response (Table 1 ).

All eyes implanted with Elvax pellets demonstrated minimal or no inflammatory response, as reflected by lack of corneal oedema or absence of hypopyon in the anterior chamber. Limbal hyperaemia was noted on the day following implantation. In all the rabbits responding positively to IGF-I, capillaries started to invade the corneal stroma 3 to 4 days following implantation and developed into a brush border that reached the pellet within 8 to 10 days. The brush border later developed into 2 or 3 main vascular trunks (Fig.1B). The new vessel growth occurred at an average rate of $0.15 \mathrm{~mm} /$ day. All eyes considered in this study showed a clear cornea, without oedema and no hypopyon in the anterior chamber. However, confirmation that the IGF-I pellet and not invasion by polymorphonuclear cells and activated macrophages was the source of angiogenic factors had to be established. Therefore, histologic studies of all IGF-I-implanted eyes demonstrating neovascularization were performed at intervals varying from 4 to 21 days. In all cases studied at days $4,7,14$, and 21 polymorphonuclear cells were absent from the corneal stroma. In all eyes studied at days 7,14 , and 21 , polymorphonuclear cells were ab- 

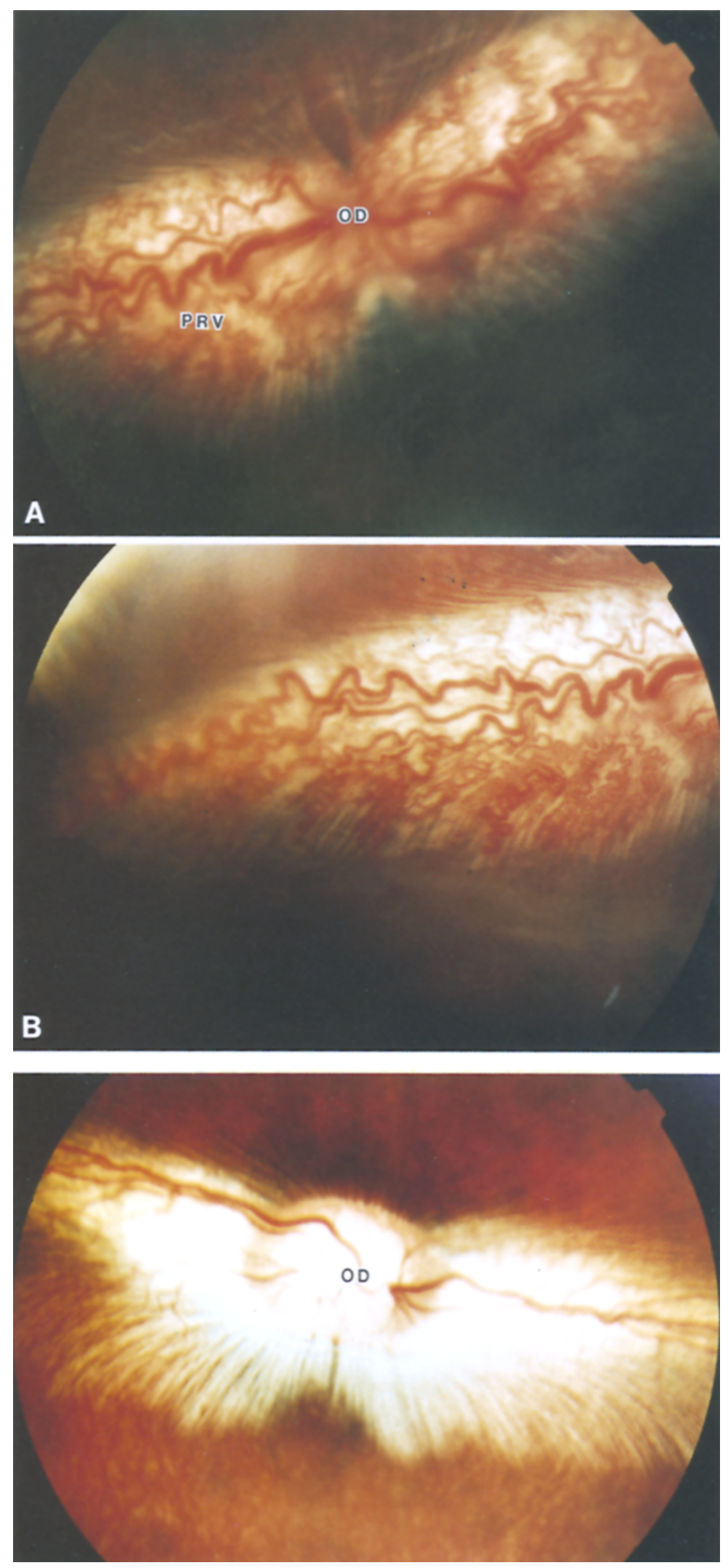

Fig. 2. A Rabbit fundus 7 days following intravitreal injection of balanced salt solution with $600 \mu \mathrm{g}$ of insulin-like growth factor $I$. The vasculature is engorged. There is marked tortuosity of the vessels, hyperaemia surrounding the optic disc (OD) and areas of distended pre-retinal vessels (PRV).

B Same area of pre-retinal vessels shown in $\mathbf{A}$, note prominent vascular changes along the lateral aspect of the medullary rays
Fig.3. A control rabbit fundus 7 days following intravitreal injection of balanced salt solution containing $600 \mu \mathrm{g}$ bovine serum albumin. The retinal vessels are confined to the two wing-shaped areas of myelinated nerve fibers, medullary rays, which extend horizontally from the optic disc (OD). The remainder of the retina is avascular sent around the corneal pocket where the dense region of capillaries existed. All five corneas implanted with $\mathrm{PGE}_{2}$ $(1 \mu \mathrm{g} / \mathrm{pellet}$ ) demonstrated a positive response (data not shown).
There was concern about whether the IGF-I was being released from the Elvax pellets because the concentrations of mitogen used were 100 - to 1000 -fold higher than those required in vitro to induce a chemotactic effect [10]. There- 

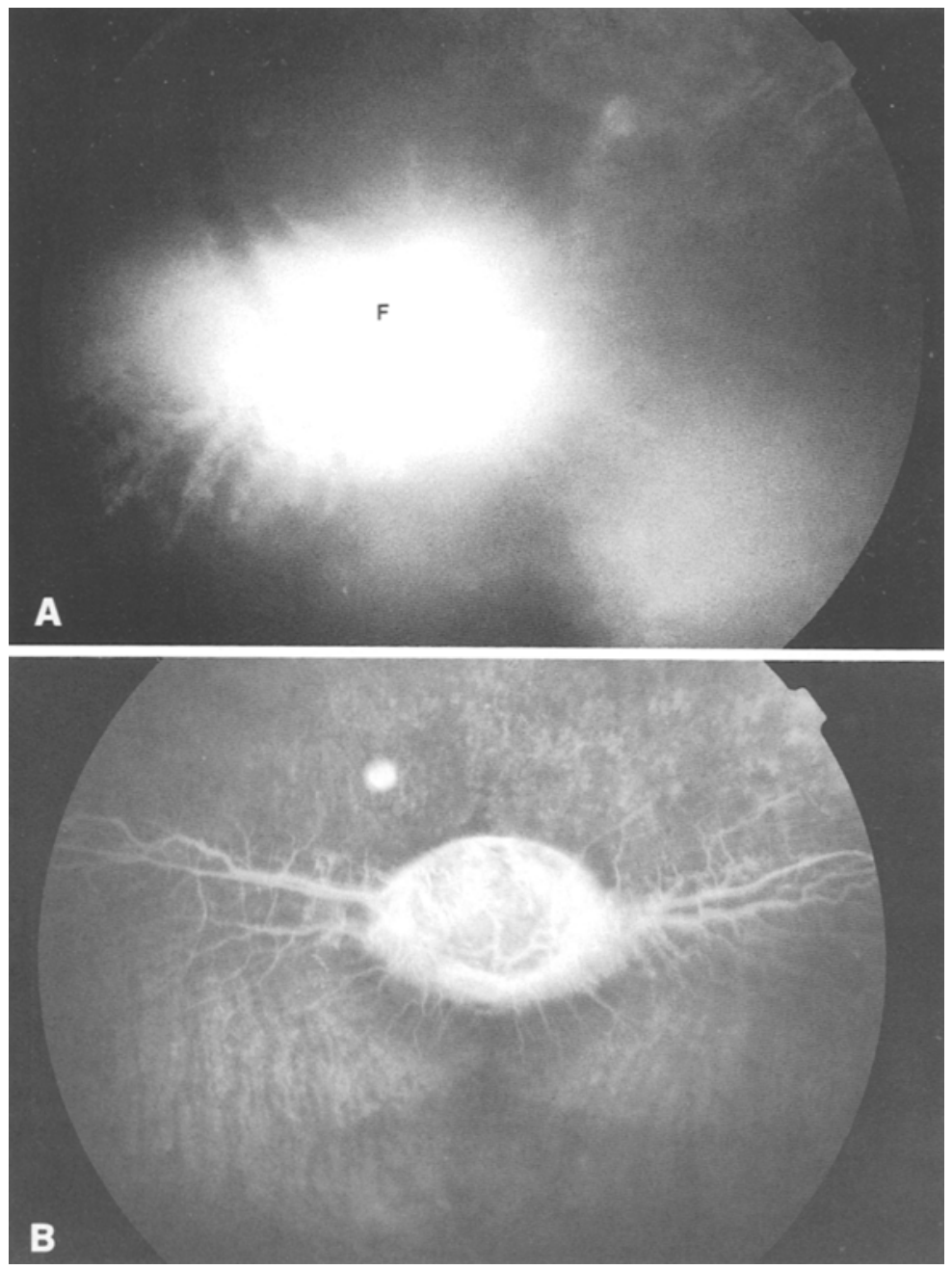

Fig.4. A Late-phase fluorescein angiogram performed on an insulin-like growth factor I-injected rabbit on day 7. The fluorescein pattern reveals increased fluorescence $(F)$ obscuring the optic disc margins and profuse late leakage. This is consistent with disruption of the blood-retinal barrier.

B Late-phase fluorescein angiogram in a control eye performed on day 7 . This photograph demonstrates normal dissipation of fluorescein with decreasing optic disc fluorescence fore, we studied the rate of release of ${ }^{125}$ I-IGF-I from Elvax pellets containing $10 \mu \mathrm{g}$ of IGF-I $(n=3)$. Following an initial burst of release of the mitogenic agent from the polymer, the IGF-I was then released from the pellet in minute concentrations at a sustained rate that lasted 4 weeks (data not shown). To further support these observations, five rabbits were not enucleated by week 3 and were observed for an additional 4 weeks. All demonstrated regression of their vascular trunks during this period.

Anatomical changes with intravitreal injections of IGF-I and $b-F G F$. The anatomical changes seen in the IGF-Iinjected eyes are shown in Figure 2A, B. Ophthalmoscopy revealed hyperaemia with vascular engorgement in all rabbits 4 days after intravitreal IGF-I injection. Vascular tortuosity, intraretinal haemorrhage, and increased hyperaemia surrounding the optic disc were noted by day 8 in all rabbits. Fibrosis developed over the optic disc and medullary ray by day 21 in all rabbits. The fundus of control eyes remained normal in appearance. Figure 3 illustrates a representative fundus from a control animal.

Fluorescein angiograms were performed in all animals receiving intravitreal IGF-I. The blood-retinal barrier, normally impervious to fluorescein, consistently demonstrated late-phase fluorescein leakage (Fig. 4 A), indicative of altered permeability of the blood-retinal barrier in the IGF-I-treated eyes. There was no leakage of fluorescein in control eyes (Fig. 4B).

All three animals injected with intravitreal b-FGF developed dense posterior subcapsular cataracts and vitreous opacification within 1 week of injection, thereby not allowing funduscopic observation of retinal changes.

Fig. 5A-F. Microscopic findings with intravitreal injection of bovine serum albumin (BSA), insulin-like growth factor-I and basic fibroblast growth factor. A Note normal distribution of vessels and scant basement membrane of eye injected with BSA (Magnification $\times 300$ ). B Note following intravitreal injection of insulin-like growth factor-I oedematous endothelial cells of vessel shown in centre of photograph, cellular proliferation and extensive extracellular matrix $(\mathrm{ECM})$ (Magnification $\times 300$ ). C Note the absence of lymphocytes but the extensive fibrosis $(F)$ and large quantities of extracellular matrix (ECM) of eye injected with basic fibroblast growth factor. D Large preretinal vessel with thin flattened endothelial cells and normal basement membrane overlaying optic nerve head $(\mathrm{ONH})$ (Magnification $\times 1450$ ) of eye injected with BSA. E Large preretinal vessel overlying optic nerve head (not shown). Note plump endothelial cells (arrowheads) surrounding lumen (L). Note prominent cellular proliferation including macrophages (M) with extensive extracellular matrix (ECM) production of eye injected with insulin-like growth factor-I (Magnification $\times 1450$ ). F Note extensive extracellular matrix (ECM) with fibroblast (F) proliferation overlying optic nerve head $(\mathrm{ONH})$ of eye injected with basic fibroblast growth factor (Magnification $\times 1450$ ) 


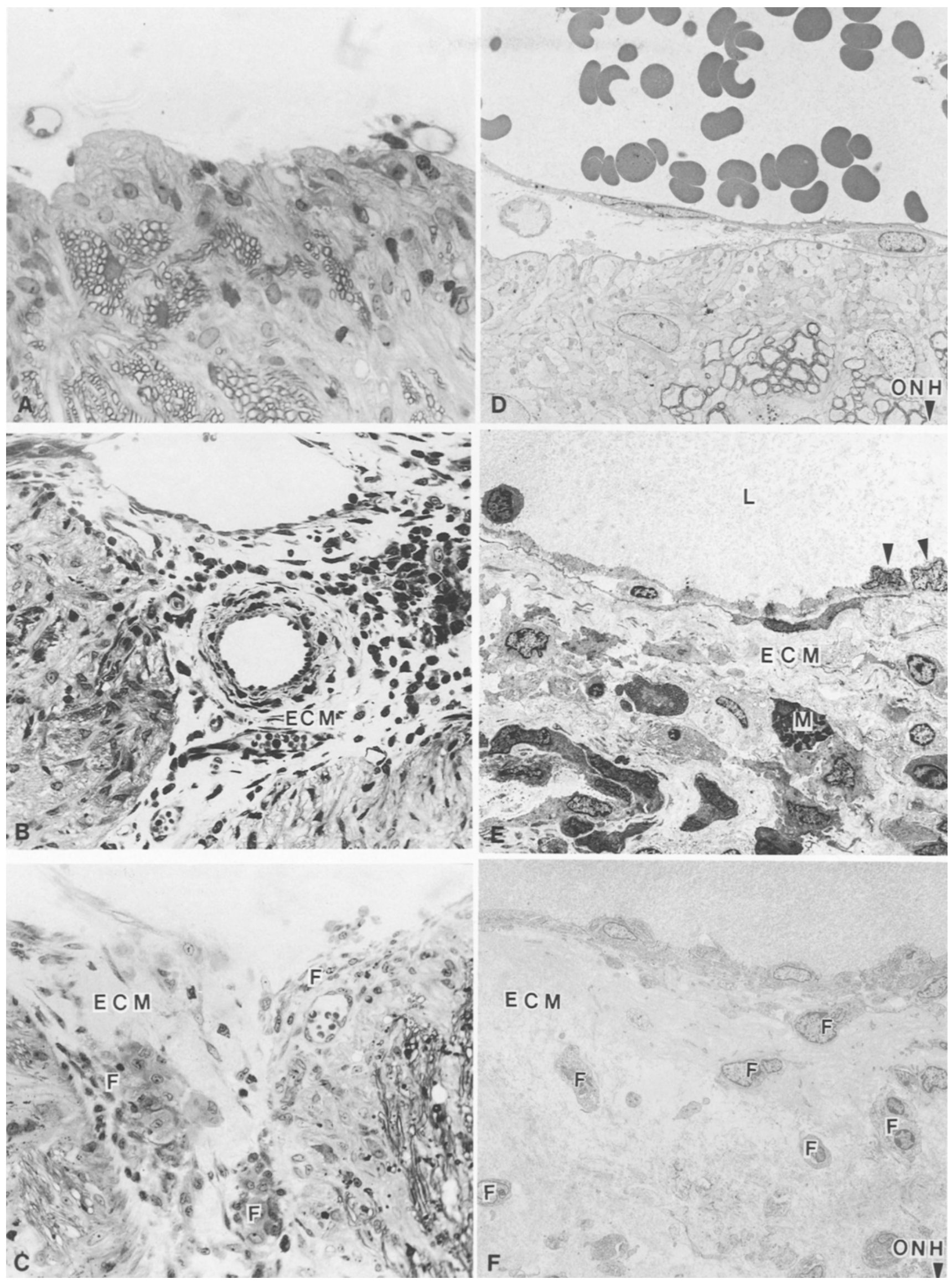


Microscopic findings with intravitreal injections of IGF-I and $b$-FGF (Fig. 5). Histological sections of control eyes injected with balanced salt solution showed sparse vessels with minimal extracellular matrix (Fig. $5 \mathrm{~A}$ ). The reaction in eyes injected with growth factors was most prominent in the area of the optic nerve head and the immediately adjacent retina. There were large abnormal vessels with oedematous endothelial cells and extensive proliferation of the extracellular matrix in retinas injected with IGF-I (Fig. 5B). Eyes injected with b-FGF developed retinal vascular changes and extensive fibrosis as demonstrated by fibroblast and extracellular matrix proliferation (Fig. 5C). In b-FGF injected eyes retinal traction and detachment adjacent to the disc was seen.

Transmission electron micrographs taken from the same areas as described for the histological sections showed vessels with thin, flat endothelial cells and minimal extracellular matrix (Fig. 5D) in eyes injected with balanced salt solution. Retinal endothelial cells of eyes injected with IGF-I were oedematous with increased endoplasmic reticulum. There was endothelial cellular proliferation, macrophages and extensive extracellular matrix production (Fig. 5 E). Eyes injected with b-FGF had proliferation of endothelial cells but the proliferation of fibroblasts and the excessive production of extracellular matrix was most noticeable (Fig. 5F).

Studies using PPC containing ${ }^{125} \mathrm{I}-\mathrm{IGF}-\mathrm{I}$. Clearance studies were performed to obtain kinetic information on the behaviour of ${ }^{125} \mathrm{I}-\mathrm{IGF}-\mathrm{I}$ in the PPC. These PPC were filled with an emulsion of collagen and $500 \mathrm{ng}$ of ${ }^{125} \mathrm{I}-\mathrm{IGF}$ I. The clearance of ${ }^{125}$ I-IGF-I occurred in two phases: the first, an initial rapid clearance (presumably diffusion of free growth factor out of the chamber) and a second, slower phase (reflecting clearance of growth factor associated with the collagen matrix or polyfluorotetraethylene of the chamber). At day $10,20 \pm 5 \%$ of the counts remained in the chambers and at day $21,5 \pm 2 \%$ of counts remained in the chamber, suggesting that these chambers could deliver physiologic amounts of IGF-I for a sustained period of at least 3 weeks.

Microscopy of retinas implanted with PPC containing $I G F-I$ or $b-F G F$. For these studies, PPC were filled with an emulsion of collagen and either 500 ng of IGF-I or b-FGF. Similar chambers containing only collagen were placed in control eyes. Funduscopic visualization of the eyes implanted with PPC chambers, containing either collagen alone, b-FGF or IGF-I, demonstrated no gross fundus changes as was seen with intravitreal IGF-I. Histological sections through the optic nerve head and adjacent retina showed normal morphology in eyes implanted with pellets containing collagen alone (Fig. 6 A). There was minimal foreign body reaction, only at the site where the pellet touched the retina. Histological sections through the optic nerve head of eyes implanted with chambers containing IGF-I (Fig. 6B) or b-FGF (Fig.6C) showed proliferation of vessels and extracellular matrix. The reaction with b-FGF was more extensive and often resulted in retinal traction and detachment.
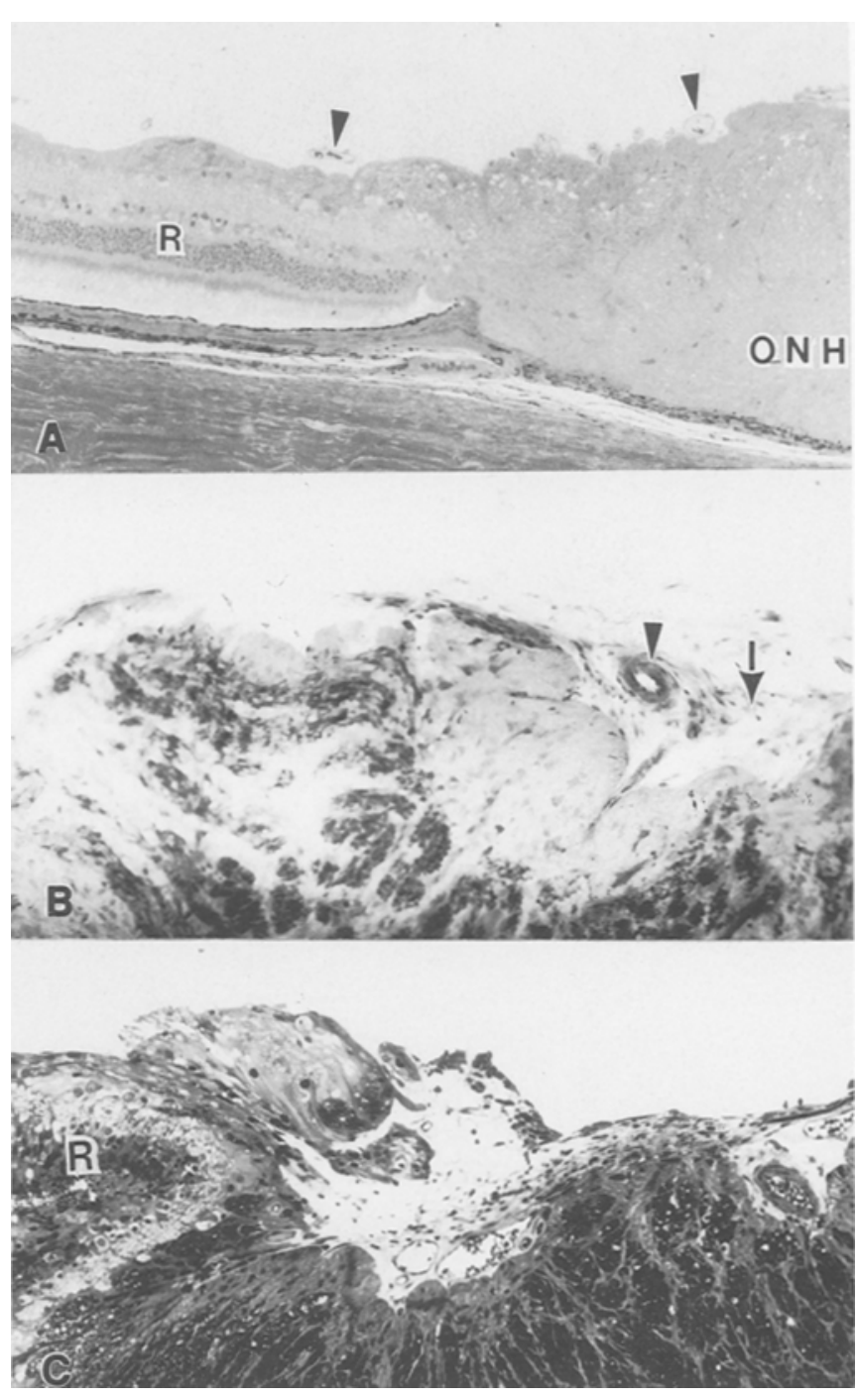

Fig. 6. A Section through the optic nerve head and adjacent retina of an eye implanted with a control pellet containing only collagen. Note the sparse thin walled vessels (arrowheads), ONH, optic nerve head, $R$, retina. (Magnification $\times 96$ ). B Section through a comparable area (as shown in $\mathbf{A}$ ) of the optic nerve head and adjacent retina of an eye implanted with a pellet containing insulin-like growth factor I. Note the thickened wall of the vessel in the optic nerve head (arrowhead) and fibrovascular proliferation on the surface of the retina (arrow). (Magnification $\times 96$ ). C Section through a comparable area (as shown in $\mathbf{A}$ ) of the optic nerve head and adjacent retina of an eye implanted with a pellet containing basic fibroblast growth factor. There is extensive fibrovascular proliferation resulting in retinal traction and detachment $(\mathrm{R})$. (Magnification $\times 96$ )

Transmission electron micrographs from retinas implanted with PPC containing collagen only showed the endothelium with normal basement membrane lining vascular channels and no evidence of inflammatory cell infiltration. Transmission electron micrographs from retinas implanted with PPC containing b-FGF showed fibroblastic proliferation surrounding vessels similar to that observed in the eyes undergoing intravitreal injections with b-FGF. Transmission electron micrographs from retinas implanted with PPC containing IGF-I showed increased numbers of perivascular spindle-shaped cells 

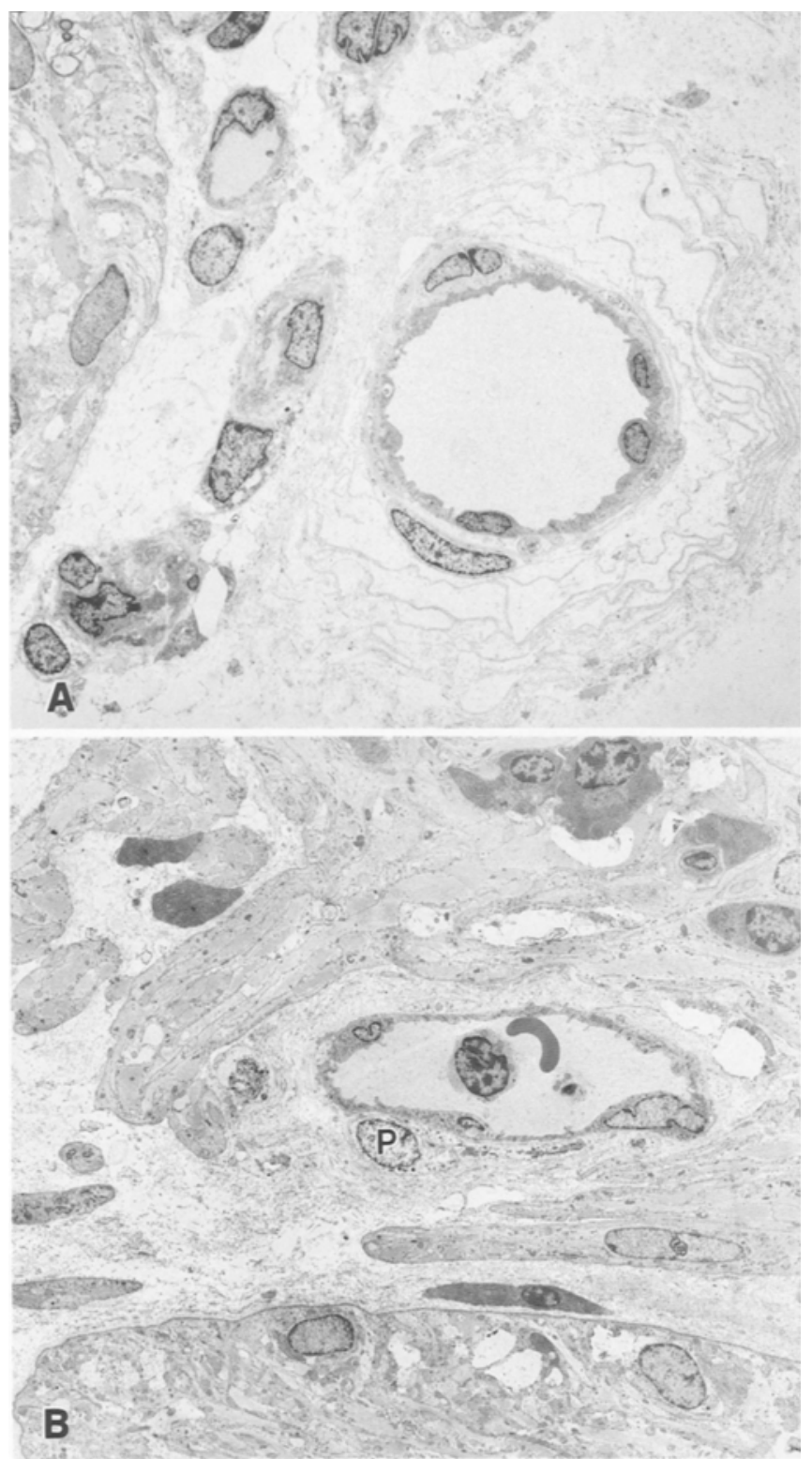

Fig. 7 A, B. Comparison of electron microscopic findings in porous polyfluorotetraethylene chambers (PPC)-containing insulin-like growth factor-I (IGF-I) and basic fibroblast growth factor. A Electron micrograph of rabbit receiving IGF-I containing PPC. Note numerous small vessels on surface of optic nerve head and reduplication of basement membrane (Magnification $\times 1350$ ). B Electron micrograph of rabbit receiving basic fibroblast growth factor containing PPC. Note vessel with reduplication of the basement membrane and necrotic pericyte $(P)$ surrounded by glial tissue (Magnification $\times 1350$ )

Fig.8 A-C. Electron microscopic findings in a rabbit which underwent placement of a porous polyfluorotetraethylene chamber (PPC) containing basic fibroblast growth factor supporting neovascularization. A Note newly-forming basement membrane (BM) and large amounts of collagen (C) around developing capillary, with well-formed tight junctions (TJ) between endothelial cells (Magnification $\times 13500$ ). B Electron micrograph showing mitotic figures $(\mathrm{MF})$ and abundant rough endoplasmic reticulum (RER) (Magnification $\times 2000$ ). C Small vessels with narrow (newly-forming) lumen with the suggestion of a tight junction (TJ) and lumen (L) forming (Magnification $\times 13500$ )

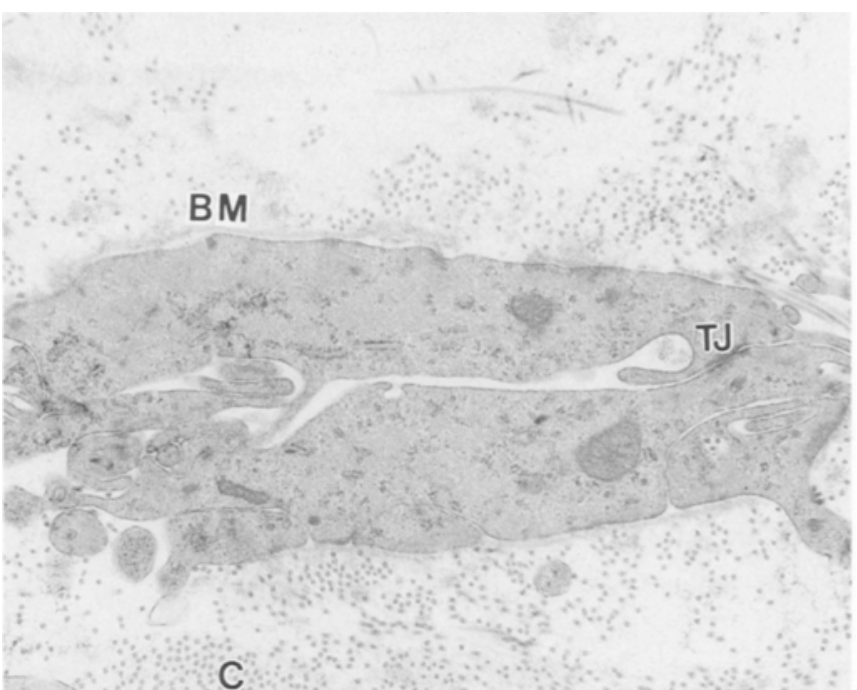

A
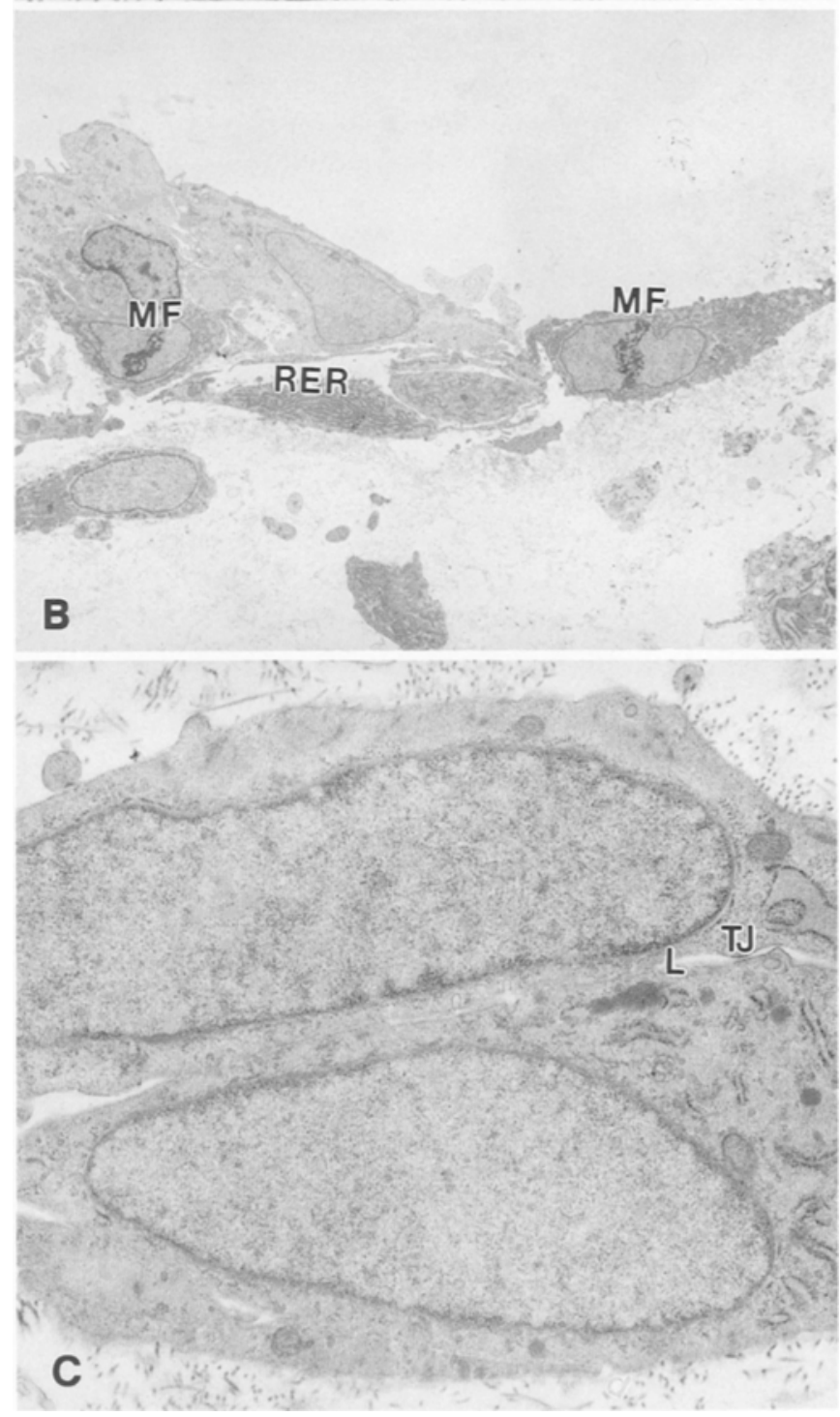
comparable to that observed in eyes receiving intravitreal injections of IGF-I. Reduplication of basement membranes, indicative of endothelial cell hyperactivity, was a consistent finding in eyes implanted with PPC containing IGF-I (Fig. 7 A) and b-FGF (Fig. 7B). There was no reduplication of basement membranes in eyes implanted with control PPC. Morphology consistent with neovascularization was observed in all retinas implanted with b-FGF and IGF-I. As shown in Figure 8, the retina from a rabbit implanted with a PPC containing b-FGF demonstrated dividing cells with newly-forming basement membranes (Fig. 8 A), nuclei with mitotic figures (Fig. 8B), and small vessels with narrow, newly-forming lumens (Fig. $8 \mathrm{C}$ ).

\section{Discussion}

Elevated IGF-I and b-FGF concentrations in the vitreous of diabetic patients with proliferative retinopathy and in vitro studies demonstrating the effect of both growth factors on endothelial cell proliferation, chemotaxis and protease production would suggest that these growth factors could modulate if not initiate retinal neovascularization. Hansson and co-workers [28] demonstrated that rapidly proliferating endothelium expresses IGF-I receptors, further supporting the significance of this growth factor in developing vasculature.

Vitrectomy fluid samples from patients with proliferative diabetic retinopathy stimulate blood vessel growth on chick chorioallantoic membranes and the proliferation of endothelial cells in culture [29]. Vitrectomy fluid from normal subjects, however, is reported to inhibit angiogenesis. Potentially competing substances in vitreous make it difficult to interpret the precise role of any growth factor in these studies $[30,31]$.

To further add to the complexity of interpreting vitreal growth factors is the presence of IGF binding proteins (IGF-BP) in the vitreous. These IGF-BP are important modulators of the biological actions of IGF-I and to date five binding proteins have been characterized [32]. Specifically vitreal IGF-BP may affect binding of IGF-I to its receptor and its biological activity or may affect the localization of IGF-I in different ocular tissues. A detailed characterization of vitreal binding proteins has been performed by Waldbillig and co-workers. Their studies support the presence of IGF-BP2, IGF-BP3 and degradation products of IGF-BP3 in bovine vitreous [33].

In the present study, three systems were used to further delineate the role of IGF-I and b-FGF as angiogenic agents and compare their respective responses. The first system used was a well-recognized method for testing the angiogenic potential of an agent. Previous studies by Risau [22] and Gospodarowitz [23] have clearly shown that b-FGF acts as an angiogenic agent in the identical system. A unique advantage of the cornea technique is that new capillary sprouts are separated from the parent vessels, an arrangement not possible with other in vivo systems [24]. In the current study we used, as developed by Langer and Folkman [24], inert pellets that released IGF-I in a sustained pattern over a 4-week period after insertion into the corneal pocket. When levels of IGF-I within the pellet were high enough $(10 \mu \mathrm{g})$ to ensure release of the growth factor from the polymer, an overwhelmingly positive response was observed, comparable to that of $b-F G F$ and the known angiogenic agent $\mathrm{PGE}_{2}$.

In the current study, we demonstrate that both IGF-I and b-FGF cause retinal fibrovascular proliferation when injected into the vitreous cavity. Intravitreal injections of IGF-I consistently had a dramatic effect on retinal vessels. Vascular engorgement and hyperaemia were seen in some rabbits as early as $24 \mathrm{~h}$ after the injection and in all rabbits 4 days after intravitreal IGF-I injection. Vascular tortuosity, haemorrhage, and hyperaemia surrounding the optic disc was a prominent feature observed in all rabbits by day 8. Disruption of the blood-retinal barrier was verified by fluorescein angiography on day 14 in selected rabbits and further suggests the presence of neovascularization as newly-formed vessels should be permeable to fluorescein. Both the IGF-I-injected eyes and the FGF-injected eyes developed retinal capillary basement membrane thickening and reduplication similar to those occurring in humans and animals with diabetes. During the second week after IGF-I and b-FGF injection, vascular changes were maintained. At 3 weeks when the experiment was terminated, the fibrosis in both b-FGF and IGF-I resulted in retinal traction and detachment.

In a similar manner the placement of PPC containing IGF-I or b-FGF on the surface of the retina resulted in the delivery of a physiologic amount of growth factor over a specific area of the retina for a prolonged period. This model system attempts to duplicate the naturally occurring events in the ischaemic diabetic retina. During the 21-day interval, no gross vascular abnormality was detected during ophthalmic examination as was seen with intravitreal IGFI, however microscopic studies at the end of the 3-week period did reveal areas of proliferation in the vicinity of the pellet, along the medullary ray and in the optic disc in eyes receiving PPC containing either IGF or b-FGF.

Our studies suggest that IGF-I and b-FGF may modulate basement membrane synthesis in such a way as to enhance endothelial proliferation and neovascularization. Basement membrane thickening and associated morphological abnormalities of the basement membrane will alter the morphological and functional state of differentiation of the overlying cells, in this case the endothelial cells [34]. Increased basement membrane thickness makes it more difficult for pericytes to contact microvascular endothelial cells; this contact is achieved through fenestrations in the basement membrane. Elimination of this contact eliminates the natural inhibition of endothelial cells by pericytes. This inhibition is provided by secretion of transforming growth factor-beta by pericytes [35].

Postnatally in mammalian species with completely vascularized retinas, endothelial cell division is rare [36]. However, under conditions of capillary non-perfusion, retinal ischaemia results in the release by retinal cells of proliferative factors [37]. Neovascularization will occur when this delicate balance is disturbed, either by activation of angiogenic factors or by a decrease in inhibitory factors. Our system attempts to upset this balance by artificially increasing one angiogenic agent at a time.

In summary, we demonstrated in vivo that IGF-I and bFGF can induce fibrovascular changes in the retina. Fun- 
dus alterations induced by these growth factors have features in common with diabetic proliferative retinopathy. Considering the limited availability of methods for inducing a breakdown in the blood-retinal barrier and vascular abnormalities similar to those seen in diabetic patients, the models described herein may prove useful for investigating the pathophysiology and potential treatments of diabetic retinopathy.

Acknowledgements. The authors thank Mr. H. Rosa for his assistance with the photographs. This study was supported by a grant (DK 18130) from the National Institute of Arthritis, Metabolism, and Digestive Diseases, and the National Eye Institute (EYO7739); and by a Peace Fellowship from the Arab Republic of Egypt.

\section{References}

1. Poulsen JE (1953) The Houssay phenomenon in man: recovery from retinopathy in a case of diabetes with Simmons disease. Diabetes 2:7-12

2. Kohner EM, Joplin GF, Cheng H, Blach RK, Fraser TR (1972) Pituitary ablation in the treatment of diabetic retinopathy. Trans Ophthalmol Soc UK 92: 79-90

3. Lundback K, Malmros R, Anderson HC (1969) Hypophysectomy for diabetic angiopathy: a controlled clinical trial. In: Goldberg MF, Fine SL (eds) Symposium on the treatment of diabetic retinopathy. Public Health Service, Publ No 1890, Washington, DC, pp 291-312

4. Tamborlane WV, Hintz RL, Bergman M, Gene LM, Felig P, Sherwin RS (1981) Insulin-infusion-pump treatment of diabetes: influence of improved metabolic control on plasma somatomedin levels. N Engl J Med 305: 303-307

5. Merimee TJ, Zapf J, Froesch ER (1983) Insulin-like growth factors: studies in diabetics with and without retinopathy. $\mathrm{N}$ Engl J Med 309:527-530

6. Dills DG, Moss SE, Klein R, Klein BEK (1991) Association of elevated IGF-I levels with increased retinopathy in late-onset diabetes. Diabetes 40: 1725-1730

7. Hyer SL, Sharp PS, Brooks RA, Burrin JM, Kohner EM (1989) A two-year follow-up study of serum insulin-like growth factor-I in diabetics with retinopathy. Metabolism 38: 586-589

8. Grant MB, Russell B, Fitzgerald C, Merimee TJ (1986) Insulinlike growth factors in vitreous: studies in controls and diabetics with neovascularization. Diabetes 35 : 416-420

9. King GL, Goodman AD, Buzney S, Moses A, Kahn CR (1985) Receptors and growth promoting effects of insulin and insulinlike growth factors on cells from bovine retinal capillaries and aorta. J Clin Invest 75: 1028-1036

10. Grant MB, Jerdan J, Merimee TJ (1987) Insulin-like growth factor I modulates endothelial cell chemotaxis. J Clin Endocrinol Metab 65: 370-371

11. Grant MB, Guay C (1991) Plasminogen activator production by human retinal endothelial cells of non-diabetic and diabetic origin. Invest Ophthalmol Vis Sci 32: 53-64

12. Bauer PI, Machoric R, Bokikg C, Sanka E, Koch SA, Horvath I (1984) Interaction of plasmin with endothelial cells. Biochem J 218: $119-124$

13. Moscatelli D, Jaffe E, Rifkin DB (1980) Tetradecanoyl pharbol acetate stimulates latent collagenase production by cultured human-endothelial cells. Cell 20: 343-351

14. Gaudric A. Falquerho F, Clement G et al. (1988) Fibroblast growth factors, transforming growth factor-beta in human vitreous from patients with proliferative diabetic retinopathy. Invest Ophthalmol Vis Sci 29 [Suppl]: 221 (Abstract)

15. Sivalingam A, Kenney J, Brown GC, Benson WE, Donoso L (1990) Basic fibroblast growth factor levels in the vitreous of patients with proliferative diabetic retinopathy. Arch Ophthalmol 108: 869-872

16. Hanneken A, deJuan E, Hutty GA, Fox GM, Schiffer S, Hjelmeland HL (1991) Altered distribution of basic fibroblast growth factor in diabetic retinopathy. Arch Ophthalmol 109: $1005-1011$

17. Gospodarowicz D (1974) Localization of a fibroblast growth factor and its effect alone and with hydrocortisone on 3T3 cell growth. Nature 249: 123-127

18. Gospodarowicz D (1976) Humoral control of cell proliferation: the role of fibroblast growth factor in regeneration, angiogenesis, wound healing and neoplastic growth. Prog Clin Biol Res 9: 1-19

19. D'Amore P, Klagsbrun M (1984) Endothelial cell mitogens derived from retina and hypothalamus: biochemical and biological similarities. J Cell Biol 99: 1545-1549

20. Herman IM, D'Amore P (1984) Capillary endothelial cell migration: loss of stress fibers in response to retinal-derived growth factor. J Muscle Res Cell Motil 5:697-709

21. Presta M, Moscatelli D, Joseph-Silverstein J, Rifkin DB (1986) Purification from a human hepatoma cell line of a basic fibroblast growth factor-like molecule that stimulates capillary endothelial cell plasminogen activator production, DNA synthesis, and migration. Mol Cell Biol 6: 4060-4066

22. Risau W (1986) Developing brain produces an angiogenic factor. Proc Natl Acad Sci USA 83: 3855-3859

23. Gospodarowitz D, Bialecki H, Thakral TK (1979) The angiogenic activity of the fibroblast and epidermal growth factor. Exp Eye Res 28: 501-514

24. Langer R, Folkman J (1976) Polymers for the sustained release of proteins and other macromolecules. Nature 263: 797-800

25. Gimbrone MA, Ramzi SC, Leapman SB, Folkman J (1974) Tumor growth and neovascularization: an experimental model using the rabbit cornea. J Natl Cancer Inst 52: 412-419

26. McDowell EM, Trump BF (1976) Histologic fixatives suitable for diagnostic light and electron microscopy. Arch Pathol Lab Med 100: 405-414

27. Reynolds ES (1963) The use of lead citrate at high pH as an electron opaque stain in electron microscopy. J Cell Biol 17: 208-212

28. Hansson HA, Brandsten C, Lossing C, Petruson K (1989) Transient expression of insulin-like growth factor: immunoreactivity by vascular cells during angiogenesis. Exp Mol Path 50:125-138

29. Chen CH, Chen SC (1980) Angiogenic activity of vitreous and retinal extract. Invest Ophthalmol Vis Sci 6: 596-602

30. Felton S, Brown G, Felberg N, Federman J (1979) Vitreous inhibition of tumor neovascularization. Arch Ophthalmol 97: $1710-1713$

31. Brem S, Preis I, Langer R, Brem H, Folkman J, Patz A (1977) Inhibition of neovascularization by an extract derived from vitreous. Am J Ophthalmol 84: 323-328

32. Rechler MM, Nissley SP (1990) Insulin-like growth factors. In: Sporn MB, Roberts AB (eds) Peptide growth factors and their receptors. Vol 95. Springer-Verlag, Heidelberg, pp 263-267

33. Arnold DR, Moshayedi P, Schoen T, Jones BE, Chader J, Waldbillig RJ (1992) Distribution of insulin like growth factor (IGF) I and II, IGF binding proteins (IGF-BP) and IGF mRNA in ocular fluids and tissues: potential sites of synthesis of IGF-BP in aqueous and vitreous. Exp Eye Res (in press)

34. Engerman RL, Pfaffenback D, Davis MD (1967) Cell turnover of capillaries. Lab Invest 17: 738-743

35. Orlidge A, D'Amore PA (1987) Inhibition of capillary endothelial cells growth by pericytes and smooth muscle cells. J Cell Biol 105: $1455-1462$

36. Ashton N (1961) Neovascularization in ocular disease. Trans Ophthalmo Soc (UK) 81: 145-161

37. Antonelli-Orlidge A, Saunders KB, Smith SR, D'Amore PA (1989) An activated form of transforming growth factor beta is produced by cocultures of endothelial cells and pericytes. Proc Natl Acad Sci USA 86: 4544-4548

Received: 28 August 1992

and in revised form: 16 November 1992

Dr. M.B. Grant, University of Florida, Department of Medicine

Division of Endocrinology and Metabolism,

P.O.Box 100226 JHMHC, Gainesville,

FL 32610-0226, USA 